\title{
Síndrome de DRESS asociado a Levetiracetam
}

\author{
A case of DRESS Syndrome associated to Levetiracetam
}

\author{
Moreno Díaz J, Martínez Huguet C, Arazo Garcés P \\ Unidad de Enfermedades Infecciosas. Hospital Universitario Miguel Servet. Zaragoza
}

\begin{abstract}
Resumen
El síndrome de DRESS es una toxicodermia asociada a eosinofilia con síntomas sistémicos. Presentamos un caso de dicho síndrome tras inicio de Levetiracetam con buena evolución tras inicio de corticoides y retirada del fármaco. Este síndrome, poco conocido y probablemente infradiagnosticado, puede ser potencialmente letal si no se trata de forma precoz.

Palabras clave: Fiebre. Rash. Eosinofilia. Levetiracetam.
\end{abstract}

\section{Introducción}

El síndrome de DRESS, acrónimo de Drug Reaction with Eosinophilia and Systemic Symptoms ${ }^{1}$, es una entidad rara caracterizada por una toxicodermia grave asociada a eosinofilia y síntomas sistémicos secundario a fármacos.

La patogenia es desconocida, proponiéndose desde reacciones inmunes mediadas por linfocitos $T$ a reactivaciones de Virus Herpes Humano tipo 62.

Presentamos un caso de este síndrome asociado a tratamiento con Levetiracetam.

\section{Caso clínico}

Paciente varón de 56 años sin antecedentes personales de interés. En el mes de Junio de 2015 presentó cuadro de mal estar general e ictericia asociado a enolismo prolongado. Ingresó en el Servido de Aparato Digestivo donde presentó crisis comicial, interpretada como crisis por deprivación, siéndole iniciado Levetiracetam durante el ingreso. Tras mejoría de síntomas y no presentar nuevas crisis comiciales así como habérsele normalizado valores analíticos, es dado de alta con el diagnóstico de Hepatitis Aguda Enólica sin criterios de cirrosis hepática.

En Agosto de 2015 el paciente presenta cuadro de fiebre de $38^{\circ} \mathrm{C}$ y exantema generalizado eritematoso, no pruriginoso, que predomina en extremidades superiores con afectación palmoplantar y periocular, así como con lesiones a nivel peribucal; en la imagen se aprecia lesiones en antebrazo y palma de mano derecha (Imagen 1), así como peribucales (Imagen2). Queda ingresado en la Unidad de Enfermedades Infecciosas para estudio de fiebre con reacción exantemática.

En la analítica realizada al paciente se objetivó eosinofilia (1400/ $\mathrm{dL}$ ) así como aumento global de transaminasas, en especial de GGT y Fosfatasa Alcalina (775 UI/L y 620 UI/L respectivamente), bilirrubina total $4,2 \mathrm{mgr} / \mathrm{dL}$ y Actividad de Protrombina de $46 \%$.

Tas comprobar estos resultados y presentar el paciente criterios tanto clínicos como analíticos de Síndrome de DRESS ${ }^{3}$, se retiró el tratamiento anticonvulsivante y se inició tratamiento con corticoides intravenosos.

A las 48 horas el paciente queda asintomático, habiendo desaparecido fiebre y exantema, quedando únicamente lesiones cutáneas

\begin{abstract}
DRESS syndrome is a toxicoderma associated to eosinophilia and systemic symptoms. We report a case of DRESS Syndrome after starting Levetiracetam with good evolution after drug withdrawal and corticosteroid therapy. This Syndrome, little know and underdiagnosed, can be lethal if not treated promply.
\end{abstract}

Keywords: Fever. Rash. Eosinophilia. Levetiracetam.

descamativas en resolución. secuenciación a corticoides orales, el paciente es dado de alta con normalización de todas las alteraciones analíticas que presentaba al ingreso.

\section{Discusión}

Es un síndrome potencialmente letal que incluye una reacción cutánea grave que puede involucrar órganos internos. Suele asociarse a la toma de fármacos anticonvulsivantes (fundamentalmente compuestos por anillos aromáticos como fenitoína), sulfamidas o alopurinol.

La incidencia estimada oscila entre 1/1000 a 1/10000 exposiciones farmacológicas ${ }^{4}$; el inicio promedio de síntomas es de 2 a 6 semanas después de haber administrado el fármaco responsable.

La fisiopatología de este síndrome es desconocida, habiéndose propuesto varias teorías tales como tales como la predisposición genética, defectos de eliminación del fármaco empleado, alteraciones inmunológicas e infecciones o reactivaciones virales. A continuación se exponen las teorías más aceptadas:

1. Deficiencia genética de enzimas detoxificantes hepáticas, produciéndose un acúmulo de metabolitos. Forman macromoléculas que producen la activación de eosinófilos y de Linfocitos T con aumento de expresión de Interleucina 5 .

2. Asociación genética entre hipersensibilidad a fármacos y HLA. Éste es el caso del HLA-B*1502, relacionado con Síndrome de Stevens-Johnson en pacientes tratados con carbamacepina.

3. Hipersensibilidad a fármacos asociada a reactivación viral. Se han descrito reactivaciones de Citomegalovirus, VHH-6, VHH-7 y Virus Ebstein-Barr coincidiendo con síntomas de hipersensibilidad a fármacos como Lamotrigina 0 Carbamacepina.

Si bien se establecen unos criterios diagnósticos estrictos (sospecha de reacción medicamentosa, eosinofilia >1500/ dL y/o linfocitos atípicos en sangre periférica, y afectación 
FIG 1: Afectación palmar y en muslo

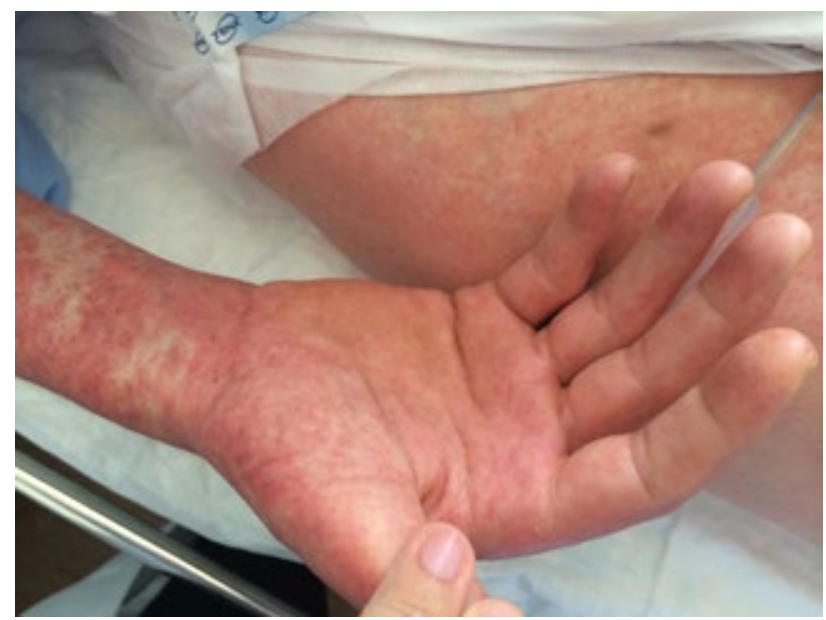

Fig 2: Descamación peribucal

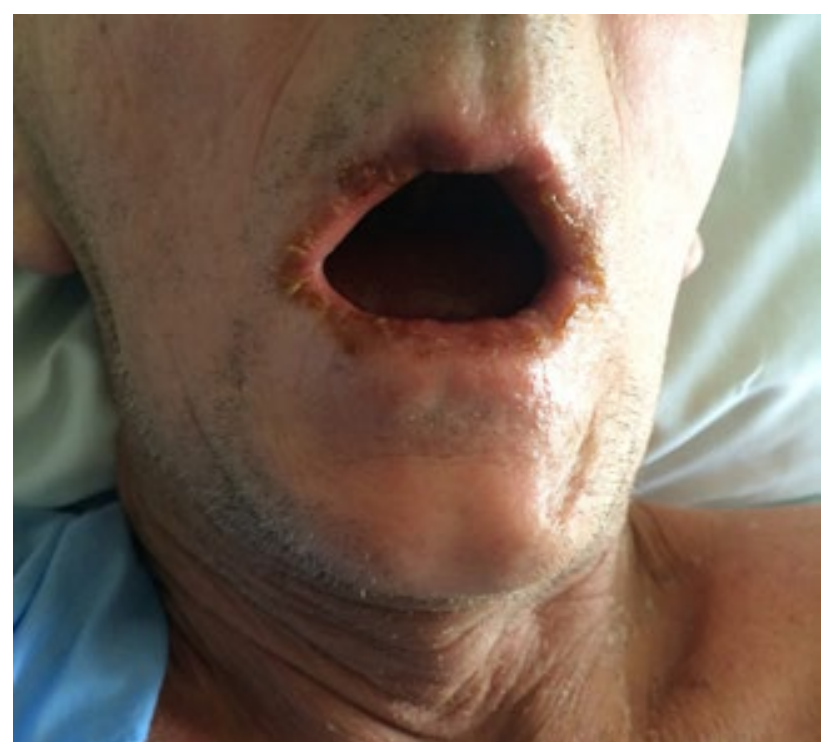

de dos 0 más órganos incluyendo la piel), siendo necesaria la presencia de los tres, se utiliza el score RegiSCAR ${ }^{6}$ el cual establece grados de probabilidad según la clínica y alteraciones analíticas del paciente (presencia de fiebre, linfoadenomegalia, grado de eosinofilia, porcentaje de rash, afectación de órganos y ausencia de otras causas) (Tabla 1). Las manifestaciones sistémicas pueden afectar varios órganos; el hígado es el más frecuentemente afectado pudiéndose encontrar desde alteraciones de las transaminasas hasta hepatitis fulminante. El rash cutáneo va a estar presente en el $97 \%$ de los casos y afecta principalmente a tronco, cara y extremidades superiores con el involucro en más del $50 \%$ del área de superficie corporal total en el $100 \%$ de los pacientes; puede además afectar a mucosas. La evolución suele ser hacia la eritrodermia con descamación.

De manera general, los pacientes con DRESS tienen una evolución benigna tras discontinuar el fármaco responsable y comenzar a usar esteroides sistémicos puesto que reducen los síntomas de la reacción de hipersensibilidad. No obstante, la mortalidad oscila entre el 3,7 y el 10\% de los casos según las series publicadas ${ }^{7}$, fundamentalmente por complicaciones secundarias a la afectación renal, pancreática o respiratoria.

Uno de los principales problemas que plantea el síndrome es su diagnóstico precoz, ya que es la retirada inmediata del fármaco la principal medida terapéutica para evitar la progresión del daño orgánico. También se ha propuesto que altas dosis de $\mathrm{N}$-acetilcisteina son eficaces en toxicodermias secundarias a anticonvulsivantes. Otros tratamientos empleados de forma anecdótica son las inmunoglobulinas humanas, la ciclosporina, la ciclofosfamida y la talidomida.

Tabla 1

\begin{tabular}{|c|c|c|c|c|}
\hline Puntuación & -1 & 0 & 1 & 2 \\
\hline Fiebre $>38,5^{\circ} \mathrm{C}$ & No & $\mathrm{Si}$ & & \\
\hline Linfoadenomegalia & & No & $\mathrm{Si}$ & \\
\hline Eosinofilia & & & $0,7-1,499 \times 109$ & $>1,5 \times 109$ \\
\hline Eosinofilia con leucocitos menores de 4000 & & & $10-19,9 \%$ & $>20 \%$ \\
\hline Linfocitos atípicos & & No & $\mathrm{Si}$ & \\
\hline$\%$ rash cutáneo & & No & $>50 \%$ & \\
\hline Rash cutáneo compatible de DRESS & No & & $\mathrm{Si}$ & \\
\hline Biopsia compatible & No & $\mathrm{Si}$ & & \\
\hline Afectación hepática & & No & $\mathrm{Si}$ & \\
\hline Afectación renal & & No & $\mathrm{Si}$ & \\
\hline Afectación pancreática & & No & $\mathrm{Si}$ & \\
\hline Afecta otros órganos & & No & $\mathrm{Si}$ & \\
\hline Resolución >15 días & No & $\mathrm{Si}$ & & \\
\hline $\begin{array}{l}\text { Evaluación de otras causas: ANA, Hemocultivos, } \\
\text { serología positiva VHA, VHB, VHC, Clamidia, } \\
\text { Micoplasma. Ninguna positiva } 0>3 \text { negativas }\end{array}$ & & & $\mathrm{Si}$ & \\
\hline
\end{tabular}


Por tanto, creemos que ante todo paciente con reacción exantemática y fiebre, se debe establecer el diagnóstico de presunción de síndrome de DRESS. Es importante realizar una historia clínica adecuada incluyendo los fármacos y el momento del inicio de los mismos para poder determinar la causalidad del cuadro y así retirar el agente causal.

Será importante conocer la afectación sistémica así como su gravedad, iniciando de forma precoz tratamiento con corticoesteroides dado que se trata de una entidad potencialmente letal.

\section{Bibliografía}

1. Bocquet H, Bagot M, Roujeau JC. Drug-induced pseudolymphoma and drug hypersensitivity syndrome (drug rash with eosinophilia and systemic symptoms: DRESS). Semin Cutan Med Surg 1996; 15 (4): 250-7.

2. Piñana $E$, Lei $S H$, Merino $R$, Melgosa $M$, De la Vega $R$, Gonzales-Obeso $E$, et al "DRESS-Syndrome on sulfasalazine and naproxen treatment for juvenile idiopathic arthritis and reactivation of human herpesvirus 6 in a 11-year old Caucasian boy.

3. Cacoub P, Mussette P, Descamps V, Meyer O, Speirs C, Finzi L, Roujeau JC. "The DRESS Syndrome: A Literature Review". The Am J Med 2011; 124: 588-597.

4. Chiou CC, Yang LC, Hung SI, Chang YC, Kuo TT, Ho HC, et al. Clinicopathological features and prognosis of drug rash with eosinophilia and systemic symptoms: a study of 30 cases in Taiwan. J Eur Acad Dermatol Venereol 2008; 22 (9): 1044-9.

5. Choudhary S, McLeod M, Torchia D, Romanelli P. Drug Reaction with Eosinophilia and Systemic Symptoms (DRESS) Syndrome. The Journal of Clinical and Aesthetic Dermatology. 2013;6(6):31-37.

6. Kardaun SH, Sidoroff A, Valeyrie-Allanore L, et al. Variability in the clinical pattern of cutaneous side-effects of drugs with systemic symptoms: does a DRESS syndrome really exist? Br J Dermatol. 2007;156:609-611.

7. Y.-C. Chen, Y.-T. Cho, C.-Y. Chang, C.-Y. Chu. Drug reaction with eosinophilia and systemic symptoms: A drug-induced hypersensitivity syndrome with variable clinical features. Dermatologica Sinica 31 (2013) 196 e 204. 